\title{
Manejo del Embarazo Prolongado
}

\author{
Dres.: R. Cifuentes B. ${ }^{*}$ y M. González ${ }^{* *}$
}

\section{INTRODUCCION}

El embarazo prolongado o de post término, vale decir, cuando sobrepasa la semana 42 de amenorrea, se asocia con una alta incidencia de morbimortalidad perinatal si se le compara con el embarazo de término (1). Es por este incrementado riesgo perinatal que frecuentemente aparecen protocolos para su manejo tales como la inducción indiscriminada de todo embarazo a las 42 semanas, lo cual aumenta la morbimortalidad materna y fetal (2); la utilización de test hormonales simples como el estriol o el lactógeno placentario, los cuales se usan cada vez menos debido a su falta de sensibilidad y bajo valor predictivo (3); la amniocentesis para evaluar el aspecto del líquido amniótico, el cual es un procedimiento de al to riesgo y que no disminuye la morbimortalidad perinatal cuando se ha utilizado en el manejo del embarazo de posttérmino con amenorrea confiable (4).

* Profesor Asociado, Jefe Sección de Obstetricia. Facultad de Salud. Universidad del Valle. Cali. Colombia.

* Residente Tercer Año. Departamento de Ginecología y Obstetricia. Facultad de Salud. Universidad del Valle. Cali. Co-lombia. .
Debido a lo anterior, diseñamos a comienzos de 1984 un protocolo de manejo para el embarazo prolongado, basado en una metodología no invasiva, de gran confiabilidad para detectar hipoxia fetal y que estuviera al alcance de toda nuestra población, como es la prueba de tolerancia fetal a las contracciones uterinas inducidas o espontáneas (PTC o PTO) (5). Por otra parte, ese diseño nos permitió conocer confiabilidad de la PTC en el manejo del embarazo de post término, incidencia de esta patología en nuestra población, análisis de algunas variables epidemiológicas (edad, paridad), antecedentes de la misma patología en embarazos previos y presencia de factores asociados que hagan prever un mayor riesgo feto-neonatal como podría ser la presencia de meconio u otra patología asociada. Posteriormente (al finalizar el año de 1984) se introdujo en nuestro Servicio de Obstetricia, la ultrasonografía en el manejo del embarazo de post término, lo cual nos ha permitido modificar un poco el protocolo inicial.

\section{PACIENTES Y METODOS}

El trabajo se realizó en el Servicio de Obstetricia del Hospital Universitario del Valle (H.U.V.) durante el año de 1984. Se diseñó un protocolo de manejo para 
todos los pacientes que consultaran por amenorrea igual o mayor de 42 semanas consistente en: si la amenorrea era confiable y el cervix favorable se inducía el trabajo de parto (T. de P.). Con cervix desfavorable se hacía PTC semanal hasta el inicio espontáneo del T. de P. o el cervix se hiciera favorable para una inducción (Fig. 1). En caso de amenorrea no

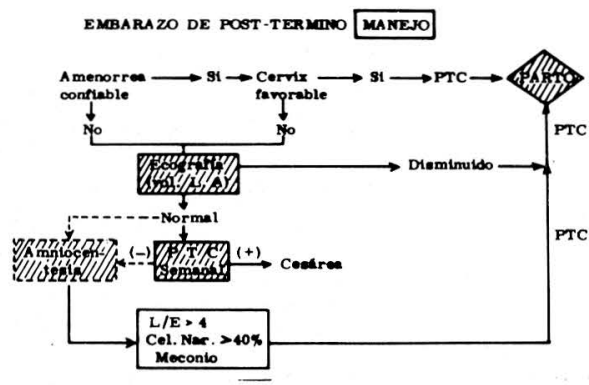

FIG. 1. Protocolo de manejo en pacientes con amenorrea igual o mayor de 42 semanas, basado en la prueba de tolerancia a las contracciones uterinas (PTC o PTO) en caso de cervix desfavorable para inducción del parto. Al finalizar el año de 1984 se introdujo en nuestro servicio la ecografía de rutina en estas pacientes, lo cual facilita aún más el manejo.

confiable se hacía amniocentesis para madurez fetal. Con feto maduro y cervix favorable se inducía el T. de P. Con feto inmaduro se reasigna la edad gestacional. Las monitorías materno-fetales fueron interpretadas por 2 ó 3 personas y se revisaron las siguientes variables maternofeto-neonatales:

- edad materna.

- antecedentes de irregularidad en los ciclos menstruales, anticoncepción hormonal o embarazo prolongado.
- edad gestacional por amenorrea.

- resultado de la monitoría fetal (PTC).

- inicio y finalización del T. de P.: espontáneo, estimulado con oxitocina, inducido o ninguno $(\sin T$. de $P$.).

- características del líquido amniótico (LA) por amniocentesis o por ruptura espontánea de las membranas.

- forma de finalización del embarazo.

- aspectos del recién nacido: Apgar, sexo, peso.

- mortalidad materna, feto-neonatal.

- patología neonatal.

\section{RESULTADOS}

Durante el período correspondiente al año de 1984 se realizaron en el Laboratorio de Medicina Perinatal del Servicio de Obstetricia del H.U.V. un total de 2.148 Pruebas de tolerancia fetal a las contracciones uterinas (PTC) (Fig. 2). De éstas, 447 (el 20.8응 fueron realizados por amenorrea prolongada lográndose el seguimiento hasta el período neonatal inmediato en 234 casos (el 52.3응. El promedio de edad de las pacientes fue de 24.8 años. El 55.5\% (130)eran primíparas y el $44.5 \%$ (104) multíparas. El promedio de edad gestacional fue de 42.7 semanas $y$ el de peso neonatal fue de $3.420 \mathrm{~g}$.

\section{Confiabilidad de la Amenorrea}

El $77 \%$ de las embarazadas tuvieron amenorrea confiable de 42 o más semanas. El $17.1 \%$ tenían el antecedente de ciclos menstruales irregulares previamente a la falla menstrual y el $4.7 \%$ de las pacientes habían utilizado anticoncepción hormonal antes del embarazo (Fig. 3). Por otra parte, el $8.6 \%$ de las embarazadas 


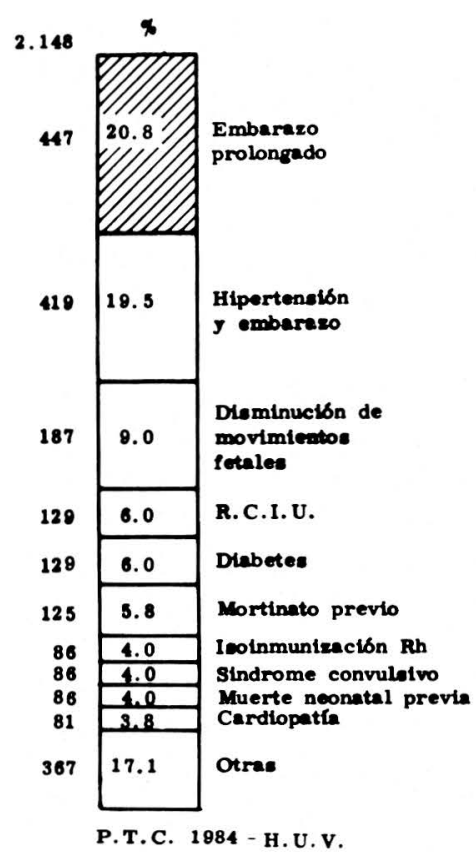

FIG. 2. De un total de 2.148 monitorías anteparto realizadas en el HUV en el año, el $20.8 \%$ fueron realizadas por amenorrea prolongada. Se muestran además las otras causas de PTC en nuestro servicio.

multíparas habían presentado la misma patología de post-madurez en una gestación previa.

\section{Evolución del Embarazo}

De acuerdo con la metodología descrita con base en la PTC hasta que se inicie $T$. de $P$. espontáneo o el cervix sea favorable para inducción del T. de P., se encontró que el $76 \%$ de las pacientes iniciaron T. de P. espontáneo; en el $14.9 \%$ se hizo inducción con oxitocina en infusión I.V. continua y el 8.9 \% no fueron a trabajo de parto (Fig. 4). No hubo fraca- so en ninguna de las 35 inducciones realizadas.

La evolución del T. de P. se muestra en la Fig. 5, en la cual se puede observar cómo el $65 \%$ de las pacientes evolucionó normalmente, el $26 \%$ hizo sufrimiento fetal agudo, en un 7\% se encontró macrosomía fetal y en el $0.8 \%$ malposición fetal (Podálica). En estos 3 últimos grupos de pacientes, el embarazo finalizó por operación cesárea.

Por otra parte, se practicó un total de 38 amniocentesis, de las cuales el

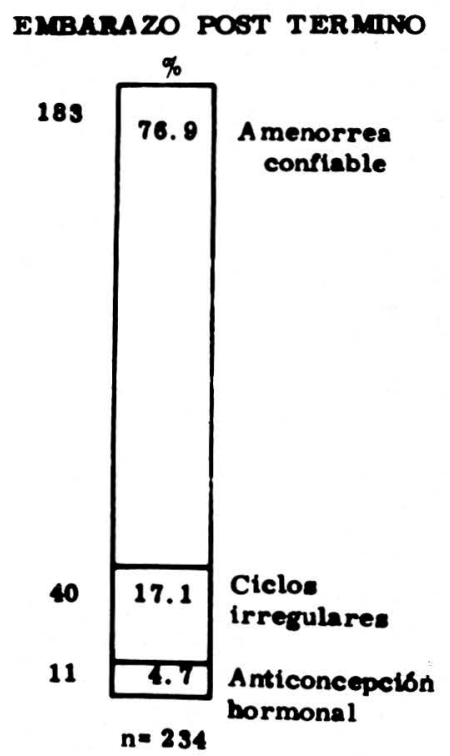

FIG. 3. Más de las tres cuartas partes de las pacientes (el 77\%) tenían amenorrea confiable. El resto tenía el antecedente de ciclos menstruales previos irregulares (17\%) o de anticoncepción hormonal previa a la gestación (el 4.7 임. 


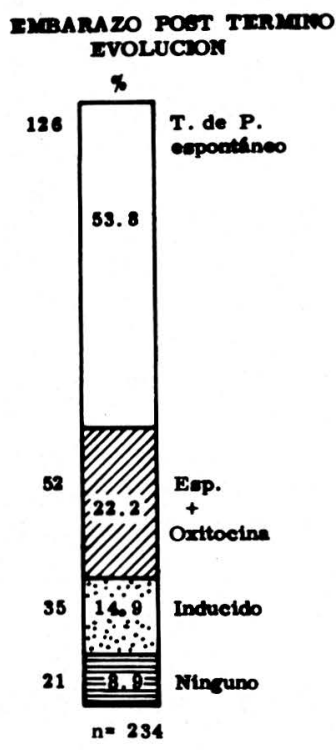

FIG. 4. Se muestra la evolución del embarazo de acuerdo con el protocolo descrito, obsérvese cómo el $76 \%$ de las pacientes inicia $T$. de $P$. espontáneo y solamente en èl $15 \%$ se necesitó inducción con oxitocina. Todas las inducciones fueron exitosas.

$100 \%$ mostró test de Clement's positivo y el $78 \%$ tenían un porcentaje de células anaranjadas mayor de 10 . En relación con la presencia de meconio en el líquido amniótico, este se encontró en el $20 \%$ de todos los embarazos, vale decir, en 4.7 pacientes. En éste grupo especial de pacientes con L.A. teñido de meconio, el $34 \%$ hizo sufrimiento fetal durante el T. de P.

\section{Aspectos del Recién Nacido}

De los 243 recién nacidos, 202 (el 86 ㅇ) tenían morfología de un recién nacido de término y solamente 41 (el 17.5) presentaba signos clínicos de postmadurez (Fig. 6). Debe mencionarse que de los 41 niños con signos de posttérmino, 37 se correspondían con una amenorrea entre 42 y 43.9 semanas y solamente 4 recién nacidos tenían amenorrea de 44 o más semanas.

La distribución de la patología neonatal se muestra en la Fig. 7, en la cual se puede observar cómo la gran mayoría de los recién nacidos (el 92\%) no tuvo ninguna patología. Hubo 4 casos de morta-

EMBARAZO POST TERMINO

EVOLUCION DEL T. DE P.

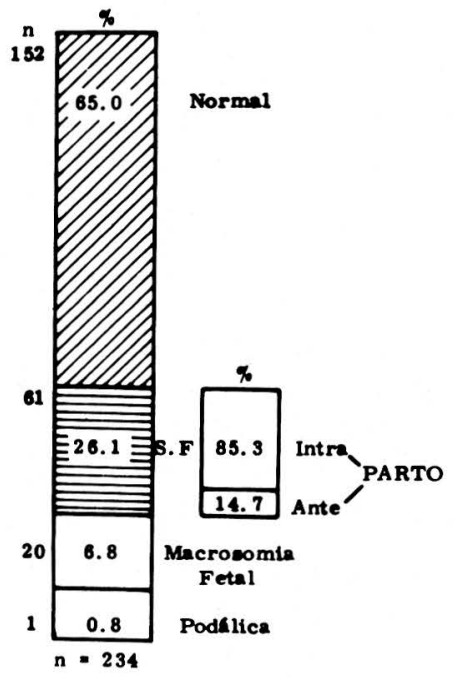

FIG. 5. Se muestra la evoluciún del T. de P. para destacar: en el $65 \%$ de las pacientes fue normal. Del $26 \%$ que hizo sufrimiento fetal, la gran mayoría (el 85 \%) fue durante el T. de P. 


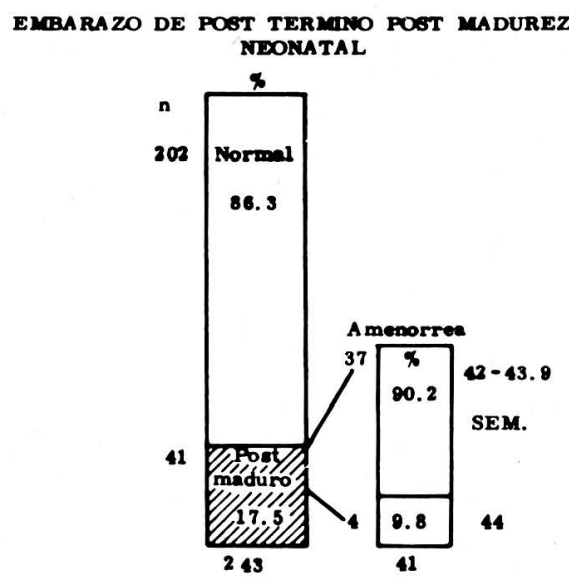

FIG. 6. De los 243 recién nacidos, solamente el $17.5 \%$ presentaba signos clínicos de post-madurez y casi todos se correspondían con amenorrea entre 42 y 43.9 semanas.

lidad, 2 intraparto (por período expulsivo prolongado en $T$ de $P$ realizado fuera del HUV) y 2 muertes neonatales (uno de ellos con mielomeningocele). Lo anterior corresponde a una mortalidad perinatal del 16 por mil.

\section{PATOLOGIA NEONATAL}

\begin{tabular}{|l|r|r|}
\cline { 2 - 3 } \multicolumn{1}{c|}{} & \multicolumn{1}{c|}{$\mathrm{n}$} & \multicolumn{1}{c|}{$\%$} \\
\hline Ninguna & 215 & 91.9 \\
Broncoaspiración de meconio & 5 & 2.1 \\
Taquipnea transitoria & 5 & 2.1 \\
Hipoxia intraparto & 4 & 1.7 \\
S.D. R. & 2 & 0.8 \\
Depresión por drogas & 1 & 0.4 \\
Malformación congénita & 1 & 0.4 \\
\hline
\end{tabular}

FIG. 7. Se muestra la patología neonatal: el $92 \%$ no tenía ninguna. Los demás recién nacidos presentaban Síndrome de Aspiración de meconio, taquipnea transitoria, síndrome de dificultad respiratoria y un caso de malformación congénita.

\section{CONCLUSIONES}

1. El 20.8 por ciento de las monitorías fetales ante parto realizadas en el HUV tienen como causa la amenorrea prolongada.

2. En el 77 por ciento de las embarazadas, la amenorrea es confiable y en el $8.6 \%$ existe el antecedente de embarazo prolongado.

3. Con el protocolo de manejo descrito por nosotros, el $75 \%$ de las embarazadas inician espontáneamente $\mathrm{T}$. de $\mathrm{P}$. En caso de inducción del mismo, no se presentó ninguna falla (se realizaron 35 inducciones con oxitocina).

4. Las evacuaciones por cesárea fueron del 35 por ciento debido principalmente a sufrimiento fetal o por desproporción feto pélvica. Este porcentaje de cesárea es similar al de la población general atendida en el HỤV.

5. Solamente el 17 por ciento de los recién nacidos presentaba signos clínicos de post madurez al examen neonatal inmediato. Se correspondían casi todos con amenorrea de 42 a 43.9 semanas.

6. Finalmente, por su baja morhimortalidad perinatal, se aconseja ia adopción del protocolo establecido por nosotros para el manejo de los embarazos con amenorrea de 42 o más semanas, en base principalmente al estado del cervix y a la evaluación del medio ambiente fetal mediante la prueba de tolerancia a las contracciones uterinas. Lo anterior evitaría inducciones innecesarias con reducción notable de la morbimortalidad materna y perinatal. 


\section{RESUMEN}

Se presenta el resultado de un protocolo de manejo para los embarazos de 42 o más semanas de amenorrea. El protocolo consiste en que si éste grupo de pacientes tenía un cervix favorable para una inducción se procedía a interrumpir el embarazo (con amenorrea confiable). Si el cervix era desfavorable - la amenorrea no confiable se seguía con prueba de tolerancia a las contracciones uterinas (PTC o PTO) hasta el inicio espontáneo del trabajo de parto ( $T$ de $P$ )

\section{BIBLIOGRAFIA}

1. BROWNE J.C.M. Post maturity. Am. J. Obstet. Cynecol 85: 573, 1963.

2. VORHERR H. Placental insufficiency in relation to postterm pregnancy and fetal post maturity. Am. J. Obstet. Gynecol 123: 67, 1975.

3. SCHNEIDER J. M., OLSON R. W.,CURET LB. Screening for fetal and neonatal risk in the post date pregnancy. Am. J. Obstet Gynecol 131: 473, 1978. o el cervix se hiciera favorable para inducir el parto. Se describen los resultados perinatales en 234 pacientes. Para destacar: 1o. El $76 \%$ hizo T. de P. espontáneo. 20. El 15\% necesitó inducción (todas exitosas). 3o. La incidencia de cesáreas fue igual a la de nuestra población general (el 35\%). 4o. Solamente el $17 \%$ de los recién nacidos presentaba signos clínicos de post madurez. 50. El $92 \%$ de los neonatos no presentaba patología. Finalmente, se aconseja la adopción de este protocolo en el manejo de ésta patología tan frecuente en nuestro medio.
4. KNOX G.E., HUDDLESTON J.F., FLOWERSC.E. Col. Management of prolonged pregnancy: Resulte of a prospective randomized trial. Am. J. Obstet. Gynecol 134: 376, 1979.

5. POSE S.V.,CASTILLO J.B., MORA ROJAS E.O., SOTO YANCES A. Test of fetal tolerance to induced contractions for the diagnosis of chronic fetal distreess. Int. J. Gynecol. Obstet. 8: 142, 1970. 